\title{
Differences in Expression of Calcium Binding Proteins in the Perirhinal and Retrosplenial Cortex of the Rat
}

\author{
Martin SALAJ ${ }^{1}$, Filip BARINKA ${ }^{1,3,4}$, Hana KUBOVÁ ${ }^{2}$, Rastislav DRUGA ${ }^{1,2}$ \\ ${ }^{1}$ Department of Anatomy, $2^{\text {nd }}$ Medical Faculty, Charles University Prague, Czech Republic, ${ }^{2}$ Institute of Physiology, \\ Czech Academy of Science Prague, Czech Republic, ${ }^{3}$ Department of Neurology, Klinik Hirslanden, Zürich, \\ Switzerland, ${ }^{4}$ Deparment of Neurology, University of Regensburg, medbo Bezirsklinikum Regensburg, Regensburg, \\ Germany
}

Received July 24, 2020

Accepted February 12, 2021

\begin{abstract}
Summary
The main aim was to describe interneuronal population expressing calcium binding proteins calretinin (CR) and parvalbumin (PV) in the perirhinal (PRC) and retrosplenial (RSC) cortex of the rat. These two cortical areas differ strikingly in their connectivity and function, which could be caused also by different structure of the interneuronal populations. Having a precise knowledge of the cellular composition of any cerebral area forms one of the basic input parameters and tenets for computational modelling of neuronal networks and for understanding some pathological conditions, like generating and spreading of epileptic activity. PRC possesses higher absolute and relative densities of $\mathrm{CR}+$ and $\mathrm{PV}+$ neurons than $\mathrm{RSC}$, but the CR : PV ratio is higher in the RSC, which is similar to the neocortex. The bipolar/bitufted neurons are most common type of $\mathrm{CR}+$ population, while the majority of $\mathrm{PV}+$ neurons show multipolar morphology. Current results indicate that main difference between analysed areas is in density of CR+ neurons, which was significantly higher in the PRC. Our results coupled with works of other authors show that there are significant differences in the interneuronal composition and distribution of heretofore seemingly similar transitional cortical areas. These results may contribute to the better understanding of the mechanism of function of this cortical region in normal and diseased states.
\end{abstract}

\section{Key words}

Interneurons • Cortex • Calretinin • Parvalbumin

\section{Corresponding author}

Martin Salaj, Department of Anatomy, $2^{\text {nd }}$ Medical Faculty, Charles University Prague, Plzeňská 311, 15006, Praha 5, Czech Republic. E-mail: martin.salaj@lfmotol.cuni.cz

\section{Introduction}

Cerebral cortex of mammals consists of two unequal neuronal populations. The larger one is formed by pyramidal neurons expressing excitatory neurotransmitter glutamate. They are projecting neurons, with long axons, whose function is to link the cortex to other cortical and subcortical regions of the brain. The second, smaller group (25-30\% of the total number of neurons) is formed by the local interneurons. They are a largely disparate group and differ in morphology, involvement, function, or expression of various neuropeptides. Most of them, however, are inhibitory, GABAergic neurons (Markram, Toledo-Rodriguez et al. 2004). Calcium binding proteins (CaBP) of calretinin $(\mathrm{CR})$, parvalbumin $(\mathrm{PV})$ and calbindin $(\mathrm{CB})$, with certain limitations, make it possible to divide cortical GABAergic interneurons into three large, nonoverlapping cell populations (Kubota, Hattori et al. 1994, del Rio and DeFelipe 1996, Gabbott and Bacon 1996, Kawaguchi and Kubota 1997, Toledo-Rodriguez, Blumenfeld et al. 2004, Toledo-Rodriguez, Goodman et al. 2005, Zaitsev, Povysheva et al. 2009). Neurons expressing calretinin $(\mathrm{CR}+)$ and parvalbumin $(\mathrm{PV}+)$ form isolated subpopulations that do not overlap. $\mathrm{CR}+$ and $\mathrm{PV}+$ interneurons differ from one another in several aspects:

- $\quad$ morphology $-\mathrm{CR}+$ neurons are typically bipolar or "bitufted"(less often multipolar), with an oval body and vertically oriented dendrites, while PV + neurons most often have multipolar morphology. 
- $\quad$ synaptic termination - the target structure of PV + interneurons are usually proximal (stem) dendrites, cellular bodies, or initial segments of the axons of pyramidal neurons. Axons $\mathrm{CR}+$ have terminations on the dendrites of surrounding neurons, but synaptic contacts prevail on the surrounding interneurons (Gabbott and Bacon 1996).

- $\quad$ electrophysiological properties - PV + interneurons belong to the group of "fast spiking" neurons. In contrast, $\mathrm{CR}+$ interneurons form a nonhomogeneous group of so-called "non-fast spiking" neurons.

- place of origin - $\mathrm{CR}+$ neurons originated in the caudal ganglional eminence, while $\mathrm{PV}+$ interneurons migrated to the developing cortex from the medial ganglional eminence (Barinka and Druga 2010, Druga 2009).

Perirhinal cortex (PRC) is the transient cortical region along the caudal half of the sulcus rhinalis. It is adjacent medially with the entorhinal cortical region (EC) and the caudal-postrhinal region (PostRH) (Brodmann 1909), which corresponds in primates with parahippocampal cortex. For these three cortical areas surrounding the hippocampal formation, Burwell uses the term "parahippocampal region" (Burwell 2000).

The PRC is further subdivided into agranular area 35 (A35) and dysgranular area 36 (A36) (Fig. 1A), (Burwell, Witter et al. 1995, Burwell 2001, Witter and Amaral 2004).

One of the most important cortical connections is the involvement of the individual regions of the PRC (A35, A36) with each other, and there are other afferent connections from the insular, pyriform and ventral temporal cortex, lateral entorhinal and postrhinal cortical region, hippocampus and subiculum. The weaker afferentation then comes from the frontal, temporal, parietal and occipital cortex. From the subcortical structures, there is the most significant association with the amygdala. There are also important inputs from olfactory cortex, ncl. endopiriformis and claustrum. Thalamic inputs come from the AM and LD nuclei, ncl. reuniens and medial geniculate body. Cortical efferentation is directed to the parietal, temporal and frontal cortex, further to the insular, postrhinal and lateral entorhinal cortical areas, as well as to the subiculum and
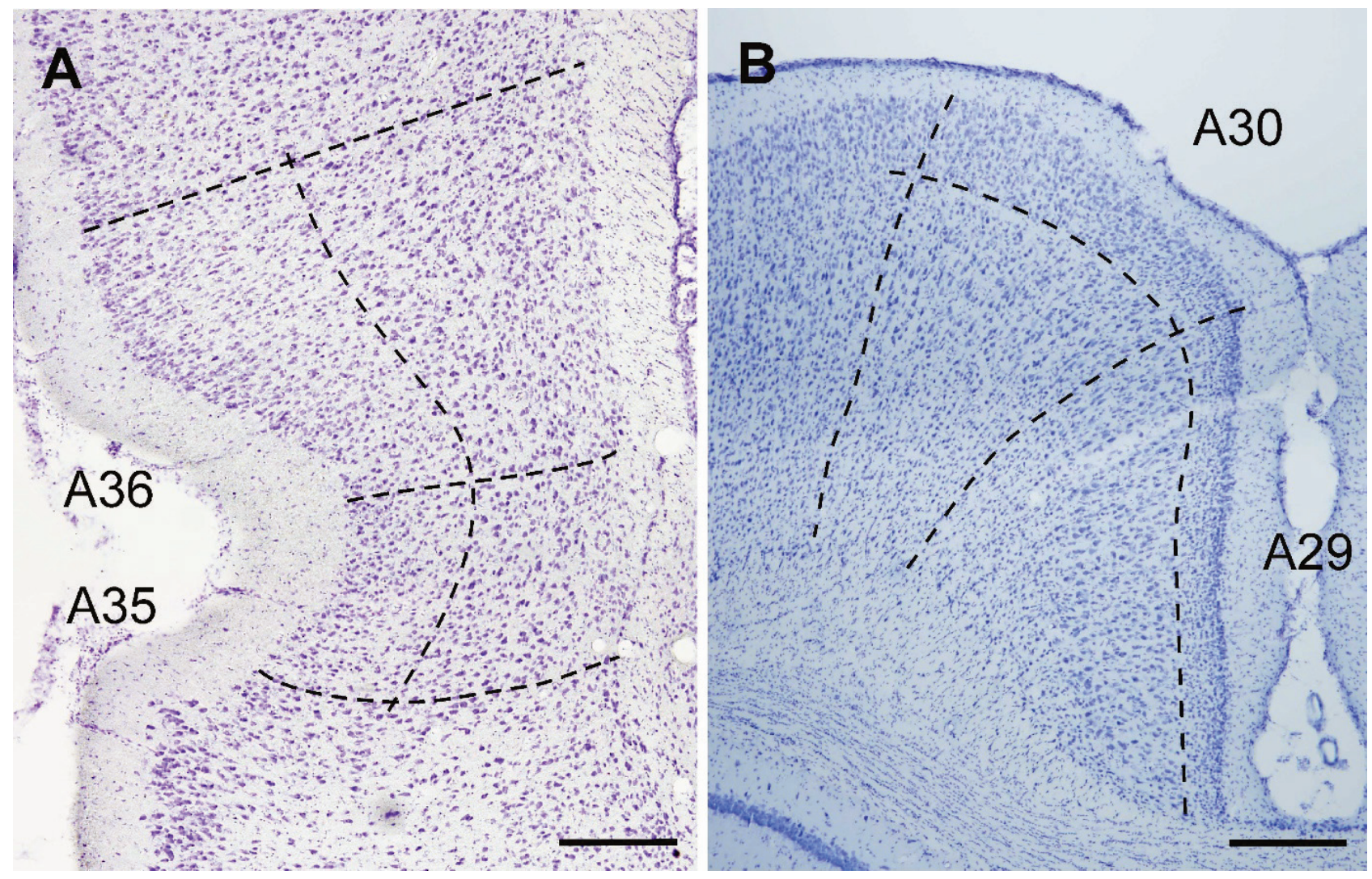

Fig. 1. Photomicrographs demonstrating cytoarchitectonics in PRC (A) and RSC (B) on Nissl-stained sections. The boundaries between individual cortical areas and between superficial (II.-IV.) and deep (V.-VI.) cortical layers are marked. Located at the level of $-4.05 \mathrm{~mm}$ (A) and $-3.55 \mathrm{~mm}(\mathrm{~B})$ anteroposteriorly from the bregma. Scale $A=200 \mu \mathrm{m}, \mathrm{B}=300 \mu \mathrm{m}$ 
hippocampus. The subcortical portion of efferent connections goes to dorsal striatum and ncl. accumbens, amygdala and periamygdalar cortex and thalamus, mainly into the LD nucleus (Burwell and Amaral 1998, Burwell and Amaral 1998, Furtak, Wei et al. 2007, Witter and Amaral 2004).

Retrosplenial cortex (RSC) is the transitional cortical area located on the mediocaudal surface of the hemisphere. It consists of Brodmann's two areas - area 29 (A29) and area 30 (A30) (Fig. 1B). Area 29 lies on the medial surface of the hemisphere, and is also referred to as granular restrosplenial cortex (RSG), whereas area 30 extends dorsolaterally from the previous region and is referred to as agranular or dysgranular retrosplenial cortex (RSA, RSD) (Brodmann 1909, Vogt 1993) Retrosplenial cortex is situated in between hippocampal formation and neocortex. It has rich afferent and efferent, ipsilateral and contralateral connections with other cortical areas and subcortical structures. Of the most important cortical connections, it is mutual connection of the individual regions and subregions of RSC (A29a, A29b, A29c, A30) to each other and further important connections from the hippocampus, subiculum and postsubiculum, the visual cortical regions A17 and A18 and the anterior cingular cortex.

From the subcortical structures, it is mainly anterior thalamus, laterodorsal thalamic nucleus (LD), claustrum and hypothalamic corpora mammillaria (van Groen and Wyss 1990, van Groen and Wyss 1992, van Groen and Wyss 2003).

The main aim was to describe interneuronal population expressing calcium binding proteins calretinin (CR) and parvalbumin (PV) in the perirhinal (PRC) and retrosplenial (RSC) cortex of the rat. These two cortical areas differ strikingly in their connectivity and function, which could be caused also by different structure of the interneuronal populations. Having a precise knowledge of the cellular composition of any cerebral area forms one of the basic input parameters and tenets for computational modelling of neuronal networks and for understanding some pathological conditions, like generating and spreading of epileptic activity.

\section{Material and Methods}

Animals

Brains of six male, previously untreated Wistar rats weighing between 350 and 400 grams were used for the present study. For the qualitative analysis of CaBP immunostaining pattern, two archival cases (males Wistar rats) were also consulted. Animals were housed under standard conditions (12 hr light / $12 \mathrm{hr}$ dark cycle, $22 \pm$ $1{ }^{\circ} \mathrm{C}$, humidity 50-60\%, free access to water and food). Experiments were approved by the Animal Care and Use Committee of the Institute of Physiology of the Academy of Sciences of the Czech Republic. Animal care and experimental procedures were conducted in accordance with the guidelines of the European Community Council directives 86/609/EEC and NIH Guidelines (Assurance No. \#A5820-01).

\section{Animals and tissue preparations}

Rats were overdosed with urethane $(2 \mathrm{~g} / \mathrm{kg}$ i.p. $)$ and perfused with $0.01 \mathrm{M}$ sodium phosphate buffer ( $\mathrm{pH}$ 7.4) followed by $4 \%$ paraformaldehyde in $0.1 \mathrm{M}$ phosphate buffer ( $\mathrm{pH}$ 7.4). The brains were removed from the skull, postfixed in buffered $4 \%$ paraformaldehyde for $3 \mathrm{hrs}$ and then cryoprotected in a gradual sucrose in $0.01 \mathrm{M}$ sodium phosphate buffer $(\mathrm{pH} 7.4)$ at $+4{ }^{\circ} \mathrm{C}$. The brains were frozen in dry ice, and sectioned in the coronal plane $(50 \mu \mathrm{m}, 1$ in- 5 series $)$ with a cryocut Leica CM 1900. Sections were stored in a cryoprotectant tissue-collecting solution (30\% ethylene glycol, $25 \%$ glycerol in $0.05 \mathrm{M}$ sodium phosphate buffer) at $-20{ }^{\circ} \mathrm{C}$ until processed. Adjacent series of sections were used for Nissl staining and immunohistochemistry.

\section{Immunohistochemical staining}

Consecutive serial coronal sections in "one of five" series were processed for 1) Nissl (cresyl violet) staining, 2) CR, 3) PV, and 4) CB immunohistochemical staining (the fifth set of sections was not used in this study). The distance between two followings sections stained with the same method was also $250 \mu \mathrm{m}$. Due to a very variable and in part very low staining intensity of $\mathrm{CB}$ immunoreactive neurons with unsatisfactory staining of dendritic tree, $\mathrm{CB}$ immunostained sections, unsuitable for quantitative analysis, were not used in the present study. For detailed description of the immunehistochemical staining see (Barinka et al. 2012, Salaj et al. 2015).

\section{Areal and laminar boundaries}

The cytoarchitectonical features and borders of the perirhinal cortical areas 35 and 36 were extensively described by Rebecca Burwell and colleagues (Burwell 2001). The Vogt classification was used to distinguish 
retrosplenial cortical areas 29 and 30 (Vogt et al. 2004).

In the rostrocaudal axis, the quantitative analysis of PRC was restricted to the area from approximately $2.75 \mathrm{~mm}$ to approximately $-7.0 \mathrm{~mm}$ relative to bregma according to (Paxinos and Watson 2007), while the quantitative analysis of retrosplenial cortex was restricted to the area from approximately $-1,8 \mathrm{~mm}$ to approximately $-6.55 \mathrm{~mm}$ relative to bregma according to (Paxinos and Watson 2007).

\section{Qualitative analysis}

The qualitative analysis was performed on immunohistochemically - and Nissl-stained sections using an image analysing software (Cell*F, Olympus) and digital camera (Olympus DP 72) attached to the microscope Olympus BX 51. The qualitative analysis was performed on both hemispheres.

\section{Quantitative analysis}

Quantitative analysis consisted of densitometric and stereological analysis and was performed on the right brain hemisphere.

\section{Densitometric analysis}

Densitometric analysis of calretinin and parvalbumin immunopositivity in cortical areas 29 and 30,35 and 36 was performed in coronal sections evenly spaced along the rostrocaudal axis. We used three coronal sections for PRC (AP -3.96 mm, AP $-5.04 \mathrm{~mm}$ a AP $6.00 \mathrm{~mm})$ and five coronal sections for RSC $(-2.05 \mathrm{~mm}$, $3.05 \mathrm{~mm},-4.05 \mathrm{~mm},-5.05$ and $-6.05 \mathrm{~mm}$ AP relative to Bregma (Paxinos and Watson 2007). In total, 18 and 30 sections (3, respectively, 5 sections from each of 6 rats) were evaluated. For detailed description of the densitometric analysis see (Barinka et al. 2012, Salaj et al. 2015).

\section{Stereological analysis}

Principles and methods of unbiased stereology as described in (West et al. 1991) were applied to achieve unbiased estimates of total neuronal density and of interneuronal densities in the studied areas. The overall neuronal density was measured on the Nissl stained sections. Neurons were counted at each second section at rostro-caudal intervals AP $-2.75 \mathrm{~mm}$ to AP $-7.0 \mathrm{~mm}$ for PRC and AP $-1.80 \mathrm{~mm}$ to $-6.55 \mathrm{~mm}$ for RSC. Neuronal numbers were measured separately in layers II-IV, V-VI and finally as a sum in layers II-VI. The cortical layer I was not included in the analysis due to the very low counts of interneurons immunostained for any of two CaBP used in the study. For detailed description of the stereological analysis see Barinka et al. (2012), Salaj et al. (2015).

\section{Statistical analysis}

Analysis of variance (ANOVA) followed by Tukey-Kramer multiple comparison test was used for the comparison of corrected relative optical densities, neuronal densities and interneuronal percentages. Probability (p) values $<0.05$ were considered significant.

\section{Results}

\section{General characteristics of immunoreactivity of calretinin and parvalbumin}

\section{Calretinin in PRC}

The total CR immunopositivity level in the PRC is markedly higher than in the adjacent entorhinal cortical region. Significant and abrupt decrease in $C R$ immunostaining on the perirhinal and entorhinal cortex transition reliably determines the ventral border of the PRC (Fig. 2A). We did not notice any significant changes in the distribution of $\mathrm{CR}$ immunopositivity along the PRC's rostro-caudal axis.

\section{Calretinin in RSC}

Unlike the perirhinal cortex, in which the CR immunopositivity of neuropile was significantly higher than in the adjacent temporal neocortex (Barinka, Salaj et al. 2012), we observed only slight differences in the total intensity of staining between RSC and surrounding neocortex. However, differences in immunoreactivity within each of the cortical layers clearly distinguish retrosplenial regions 29 and 30 from adjacent cortical arrays (Fig. 3A).

The total intensity and distribution of CR staining does not change significantly in the RSC along the rostro-caudal axis with one exception of the higher intensity of staining in the region 29 a, which is found only in the caudal sections. We did not observe any difference in staining patterns between areas $29 \mathrm{~b}$ and $29 \mathrm{c}$, previously noting that an unequivocal distinction between these two areas was not possible from the CR-immunostained sections. 

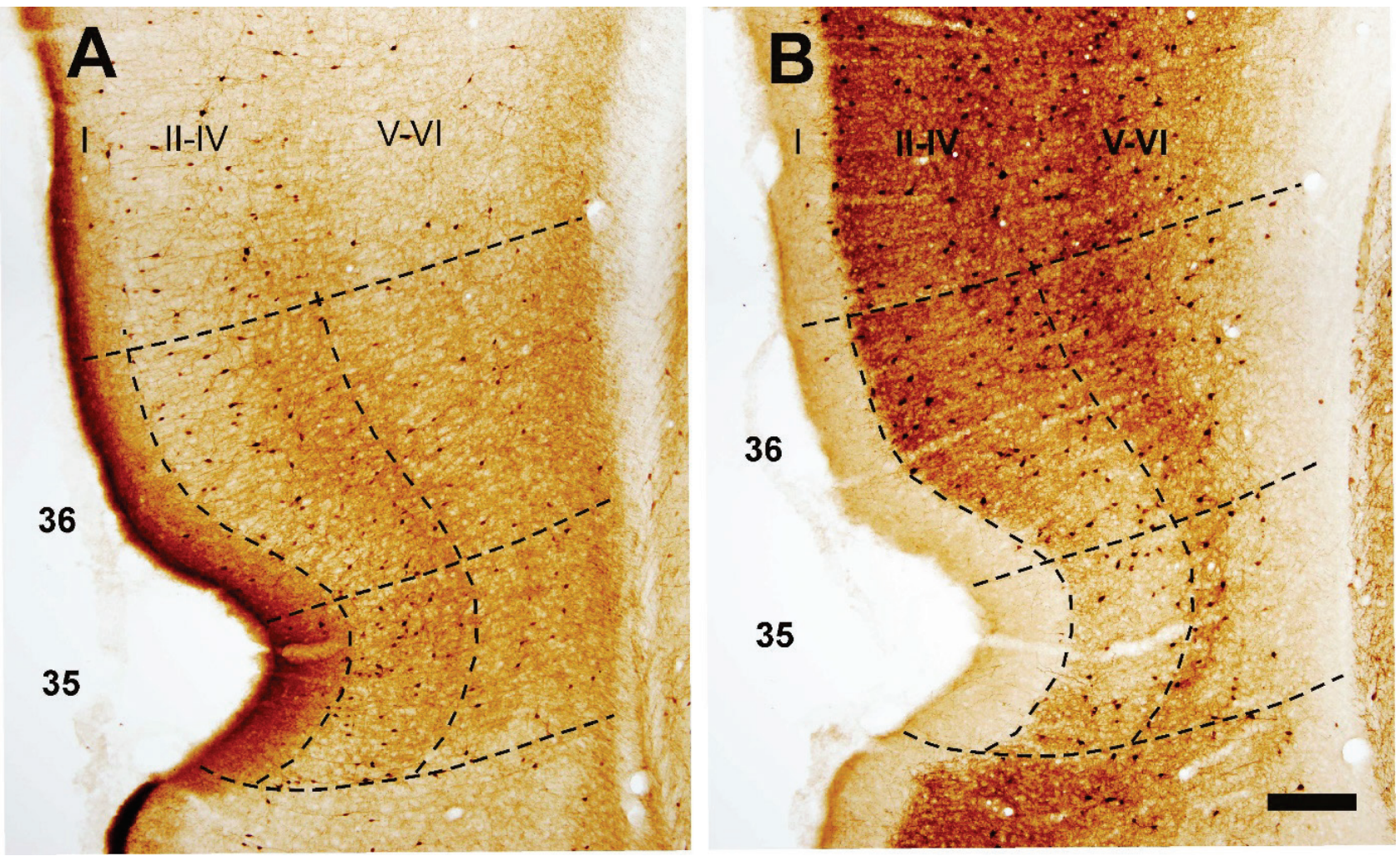

Fig. 2. Photomicrograph illustrating the distribution of calretinin (A) and parvalbumin (B) immunoreactivity in cortical areas $A 35$ and A36. Boundaries of areas and cortical layers are shown. Located at $-5.05 \mathrm{~mm}$ anteroposteriorly from the bregma. Scale $=200 \mu \mathrm{m}$.
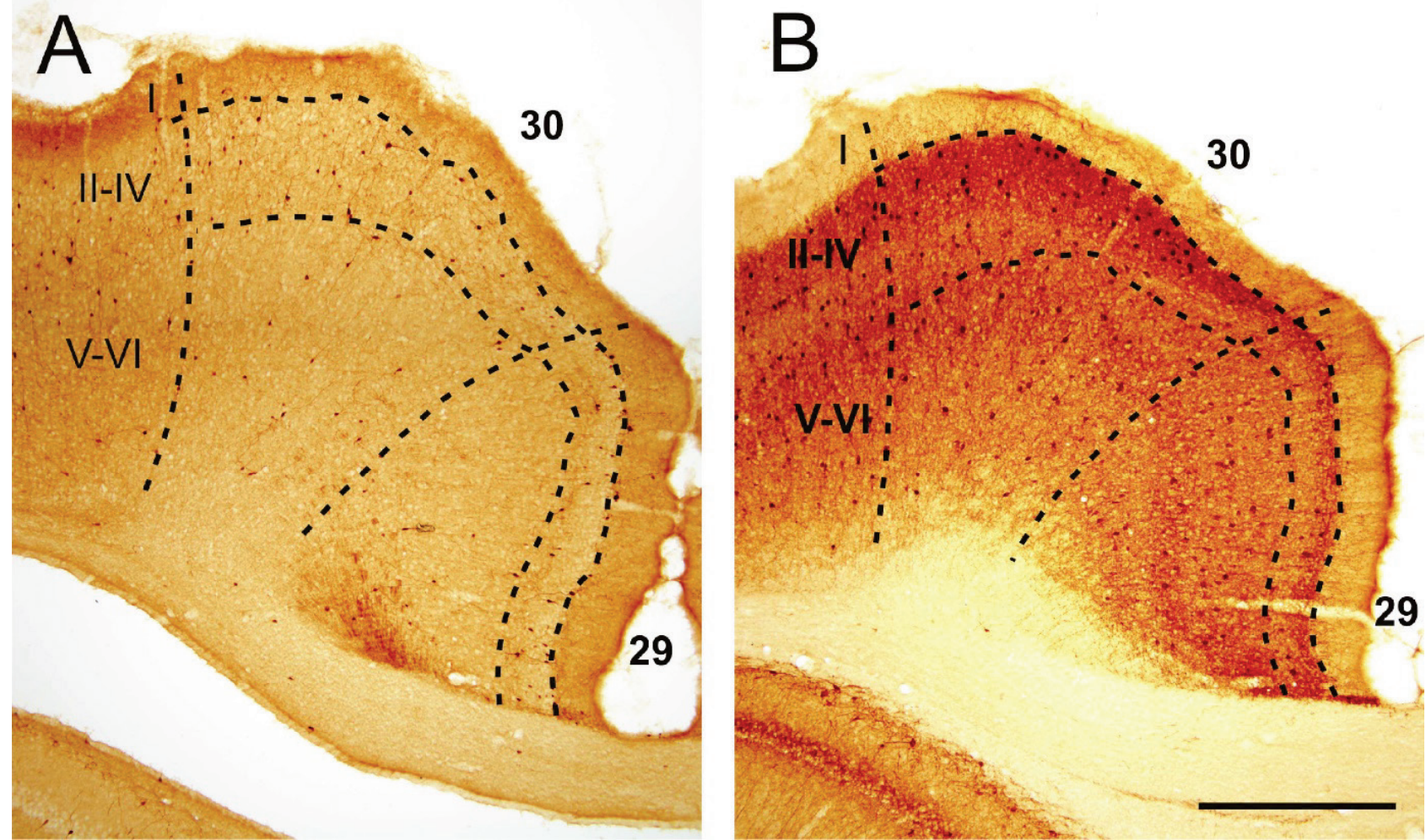

Fig. 3. Representative photomicrograph illustrating the distribution of calretinin (A) and parvalbumin (B) immunoreactivity in cortical areas A29 and A30. Boundaries of areas and cortical layers are shown. Located at the level of $-4.05 \mathrm{~mm}$ anteroposteriorly from the bregma. Scale $=500 \mu \mathrm{m}$. 


\section{Parvalbumin in PRC}

In contrast to CR-immunoreactivity, sections stained with PV are significantly lower immunopositive compared to adjacent entorhinal and temporal cortex (Fig. 2B).

\section{Parvalbumin in RSC}

On the sections stained with parvalbumin, we did not notice such significant differences in the RSC as compared to the adjacent neocortex as in the case of CR-stained material (Fig. 3B). The A29 layer I shows only a low colour intensity. Deeper we can find a band of high intensity in II. layer, sharply bounded against layer I, whose intensity gradually decreases toward III. layer. This together with layer IV. exhibit only low immunopositivity, gradually moving to higher intensity in the V. layer. Finally, we can see a lower intensity in VI. layer.

At the border between A29 and A30 there is a sudden and significant increase in the thickness of the high intensity lane in II. and III. (Fig. 3B) Thanks to these significant changes in the immunoreactivity distribution, we can clearly distinguish the boundary between A29 and A30 on PV stained sections (Fig. 3B).

\section{Neuronal morphology}

\section{Morphology of neurons in the PRC}

Generally, the somatodendritic morphology of $\mathrm{CR}+$ and $\mathrm{PV}+$ neurons in both studied areas was less distinguishable than in the surrounding neocortical regions. In all cases, the dendritic trees of neurons located in V. and VI. layer are better coloured and visible than in II. and III. layer.

\section{Calretinin}

Vertically oriented bipolar or "bitufted" neurons. The most common type of $\mathrm{CR}+$ neurons was the bipolar type. These cells formed the largest group in both the surface (II.-IV) and the deep cortical layers (V.-VI.) of both regions.

Multipolar neurons. The cell bodies of the multipolar CR + neurons were most often in the shape of a polygon, with three or four major dendrites radially withdrawing from the body and pointing all the way to the surroundings. Less often, we found inverted pyramidtype neurons.

Horizontally oriented bipolar or "bitufted" neurons. In this rare type of $\mathrm{CR}+$ neurons, 2 bundles of dendrites, or only two primary dendrites arise from the opposed poles of the fusiform body.

\section{Parvalbumin}

Vertical bipolar or "bitufted" neurons. We found this type of PV + neurons only rarely. They usually had an oval perikaryon with dendrites extending from the upper and lower poles of the cell body.

Multipolar neurons. Neurons forming the most numerous PV + type neurons in all investigated areas and layers. The cell bodies typically had a round or oval shape, often with four or more dendrites, radially branches in all directions. Dendrites were mostly thin and slightly colored.

Horizontal bipolar or "bitufted" neurons. The less frequent morphological type of PV + neurons was observed more frequently in deeper cortical layers.

\section{Morphology of neurons in RSC}

In general, neuronal morphology was better distinguishable in CR neurons than those stained on PV. The cell bodies, proximal dendrites and distal parts of the dendritic segments were recognizable in most of the CR stained neurons. An intensely stained dendritic tree as well as longer dendritic segments were more common in the V-VI layers than in the supra-granular layers. On the other hand, only PV perikarya and occasionally proximal dendrites were observed for PV stained neurons. Their distal parts of the dendritic arborisation could not be distinguished from the strongly immunopositive neuropile.

\section{Calretinin}

Vertically oriented bipolar or "bitufted" neurons. The most common type of $\mathrm{CR}+$ neurons was the bipolar type. These cells formed the largest group in the deep cortical layers (V.-VI.) of both regions.

Multipolar neurons. The multipolar neurons were the second largest group of total and most common cell type in superficial cortical layers (II.-IV) of both regions.

Horizontally oriented bipolar or "bitufted" neurons. Horizontally oriented CR + neurons formed only a small part of the total population in both studied areas.

\section{Parvalbumin}

Regarding $\mathrm{PV}+$ neurons in $\mathrm{RSC}$, the vast majority of observed neurons were distinguishable only by cell body or short segments of primary dendrites. Cell 
processes were well stained, but only exceptionally distinguished from strongly PV immunopositive neuropile. Most distinct neurons had a circular or multipolar shape. Less often, we observed in the deep layers neurons with a vertically oriented body of oval shape.

\section{Densitometric analysis of calretinin and parvalbumin immunoreactivity}

Densitometric analysis served to quantify differences in CR and PV immunoreactivity of neuropile in PRC and RSC. We measured corrected optical density (cROD) in both studied areas (see Materials and Methods). The results are summarized in Table 2.

\section{Densitometry in PRC}

On CR stained sections, the cROD in A35 (4.7 \pm 0.5$)$ was highest. Slightly but not significantly, lower values were then measured in A36 (4.4 \pm 0.6$)$. On sections stained for $\mathrm{PV}$, the cROD values were $3.7 \pm 0.4$ for A35 and 5.1 \pm 0.7 for A36, again without a statistically significant difference. To determine the possible differences in CR and PV immunopositivity along the rostro-caudal axis, we compared cRODs from three different rostro-caudal levels for all studied areas. We did not observe any statistically significant differences in the cROD values from the mentioned areas (without numerical data)

\section{Densitometry in RSC}

In $\mathrm{CR}$ stained sections, the cROD 2.1 value was in both of the areas described. In sections stained for PV, the cROD value was significantly higher, 4.5 in A29 and 4.4 in A30 (Fig. 3).

To determine possible differences in immunoreactivity for $\mathrm{CR}$ and PV along the rostro-caudal axis, we further measured the cROD from 5 different levels in both investigated areas. No statistically significant deviation in cROD values was found in all examined sections.

\section{Quantitative density analysis of immunoreactive neurons}

Densities of $C R+$ and $P V+$ neurons in A29, A30, A35 and A36 was determined stereologically and expressed as the number of neurons per $1 \mathrm{~mm}^{3}$ of the cortex \pm standard deviation (S.E.M.) alone and especially for the cortical layers II.-IV., V.-VI. and finally overall
II.-VI. The results are summarized in Table 1.

\section{- Density of $C R+$ and $P V+$ neurons in PRC}

Distribution of $\mathrm{CR}+$ neurons was similar in both studied regions, with high neuronal density in surface and significantly lower dense in deep cortical layers. We also observed differences in neuronal density between the studied areas. This value decreases along the ventrodorsal axis, with the highest value in A35 (3194 \pm 211 neurons / $\mathrm{mm}^{3}$ for II.-VI.) and a much lower value in A36 (2520 \pm 363 neurons per $\mathrm{mm}^{3}$ ) and appears to have a decreasing tendency towards dorsal (Barinka et al. 2012). This decrease is particularly pronounced in surface layers II. and III. (II-IV) (Table 1 for numerical values).

In both studied areas, PV + neurons were decomposed similarly to $\mathrm{CR}+$ neurons, with higher cell numbers in superficial cortical layers, although the difference between the individual layers was not as marked as $\mathrm{CR}+$ neurons. Unlike $\mathrm{CR}+$ neurons, $\mathrm{PV}+$ neurons were more evenly distributed among the study areas. The neuronal density in A35 was $3805 \pm 120$ neurons $/ \mathrm{mm}^{3}$ for II.-VI. layer and only slightly higher was in case of A36 $\left(4336 \pm 223\right.$ neurons $\left./ \mathrm{mm}^{3}\right)$ (Table 1).

\section{- Densities of $C R+$ and PV + neurons in RSC}

The distribution of $\mathrm{CR}+$ neurons was very similar in A29 and A30, with high density in superficial cortical layers and significantly lower density in deep layers. However, overall, the higher the density of neurons in A29 compared to A30. This difference was statistically significant.

Density of PV + neurons in V.-VI. layer was significantly higher in A29 compared to A30. Differences in the neuronal densities of the remaining areas (layers IIIV and II-VI) did not reach statistical significance.

The total neuronal density and percentages of $C R+$ and $P V+$ neurons from total neuronal density

In order to avoid a possible error in comparing the different cortical regions that could be caused by different neuronal densities in sulcus rhinalis and hemisphere convexity, we determined the total number of neurons on Nissl-stained incisions, and then we expressed relative counts of interneurons in all individual studied areas as a percentage of the total neuronal population.

\section{- Total neuronal density in PRC}

The total neuronal density was consistently higher in the superficial layers of the two studied areas. In 
comparison of both areas, both the total neuronal density in II.-IV. layer of both neuronal density individually measured for surface and deep layers higher in A35 and lower in A36. Individual values and levels of statistical significance are in Table 1.

- $\mathrm{CR}+$ interneurons form $6.4 \%$ in A35 and $5.5 \%$ in A36 of the total neuronal population II.-VI. layers (Table 1). Unlike CR + neurons, we observed only statistically insignificant differences in the percentage expression of neuronal $\mathrm{PV}+$ neuron density from the total neuronal density in the studied areas (Table 1).

- Total neuronal density in RSC

The total neuronal density (determined stereologically on Nissl stained sections) was significantly higher in the A29 surface layers compared to A30. Thanks to this striking difference in II.-IV. layer was also the total density in II.-VI. layer significantly higher in A29 vs. A30, although the overall density in
V.-VI. layer of the two studied areas was similar.

$\mathrm{CR}+$ interneurons accounted for $2.2 \%$ in A29 and $2.1 \%$ in A30 of the total population of II.-VI. layers (Table 1). In the case of PV + neurons, no significant difference was found. Of the total population II.-VI. layers consisted of PV + interneurons $4.4 \%$ in A29 and $4.5 \%$ in A30 (Table 1).

\section{Discussion}

Neocortical formations of the mammalian brain consist of two basic types of neurons. $20-30 \%$ of cortical neurons are local interneurons expressing the inhibitory neurotransmitter GABA; 70-80 \% represent excitatory (projection) glutamatergic neurons with predominant pyramidal morphology. Further classification of cortical interneurons is based on their neurochemical phenotype, their functional properties and synaptology. Expression of calcium-binding proteins has been shown to be one of the

Table 1A. Absolute neuronal densities per $\mathrm{mm}^{3}$ of cortex \pm S.E.M.

\begin{tabular}{|c|c|c|c|c|c|c|}
\hline & \multicolumn{3}{|c|}{ A29 } & \multicolumn{3}{|c|}{ A30 } \\
\hline & II.-IV. & V.-VI. & II.-VI. & II.-IV. & V.-VI. & II.-VI. \\
\hline Nissl & $145969 \pm 8304^{x}$ & $68815 \pm 2160$ & $93477 \pm 3456^{x}$ & $97484 \pm 3468$ & $67146 \pm 2265$ & $79904 \pm 1098$ \\
\hline$C R$ & $5363 \pm 165^{x}$ & $1174 \pm 30^{x}$ & $2050 \pm 54^{x}$ & $3330 \pm 221$ & $953 \pm 62$ & $1656 \pm 106$ \\
\hline \multirow[t]{3}{*}{$P V$} & $4461 \pm 223$ & $3932 \pm 310^{x}$ & $4059 \pm 282$ & $4715 \pm 247$ & $3064 \pm 206$ & $3578 \pm 196$ \\
\hline & \multicolumn{3}{|c|}{ A35 } & \multicolumn{3}{|c|}{ A36 } \\
\hline & II.-IV. & V.-VI. & II.-VI. & II.-IV. & V.-VI. & II.-VI. \\
\hline Nissl & $53803 \pm 842^{a}$ & $47716 \pm 2359$ & $50365 \pm 1257$ & $47926 \pm 863$ & $45442 \pm 1286$ & $46500 \pm 1053$ \\
\hline$C R$ & $4967 \pm 428^{\mathrm{a}}$ & $1439 \pm 90$ & $3194 \pm 211$ & $3617 \pm 296$ & $1143 \pm 85$ & $2520 \pm 363$ \\
\hline$P V$ & $4805 \pm 202$ & $2930 \pm 150$ & $3805 \pm 120$ & $5240 \pm 297$ & $3550 \pm 210$ & $4336 \pm 223$ \\
\hline
\end{tabular}

$x$ - statistically significant difference in comparison with $A 30, p<0.05, a-$ statistically significant difference in comparison with $A 36, p<0.05$.

Table 1B. Percentages of CR and PV-immunoreactive neurons in the overall neuronal population \pm S.E.M.

\begin{tabular}{|c|c|c|c|c|c|c|}
\hline & \multicolumn{3}{|c|}{ A29 } & \multicolumn{3}{|c|}{ A30 } \\
\hline & II.-IV. & V.-VI. & II.-VI. & II.-IV. & V.-VI. & II.-VI. \\
\hline$C R$ & $3.7 \pm 0.3$ & $1.7 \pm 0.1$ & $2.2 \pm 0.1$ & $3.5 \pm 0.2$ & $1.4 \pm 0.1$ & $2.1 \pm 0.1$ \\
\hline \multirow[t]{3}{*}{$P V$} & $3.1 \pm 0.3^{x}$ & $5.7 \pm 0.5$ & $4.4 \pm 0.4$ & $4.9 \pm 0.3$ & $4.6 \pm 0.3$ & $4.5 \pm 0.3$ \\
\hline & \multicolumn{3}{|c|}{ A35 } & \multicolumn{3}{|c|}{ A36 } \\
\hline & II.-IV. & V.-VI. & II.-VI. & II.-IV. & V.-VI. & II.-VI. \\
\hline$C R$ & $9.2 \pm 0.7$ & $3 \pm 0.2$ & $6.4 \pm 0.4$ & $7.6 \pm 0.7$ & $2.5 \pm 0.2$ & $5.5 \pm 0.9$ \\
\hline$P V$ & $8.9 \pm 0.4$ & $6.2 \pm 0.2$ & $7.6 \pm 0.2$ & $11 \pm 0.8$ & $7.9 \pm 0.7$ & $9.4 \pm 0.7$ \\
\hline
\end{tabular}

$x$ - statistically significant difference in comparison with $A 30, p<0.05$. 
Table 2. Corrected relative optical density \pm S.E.M. of neuropile immunopositive for CR and PV.

\begin{tabular}{ccccc}
\hline & $\mathbf{A 2 9}$ & $\mathbf{A 3 0}$ & $\mathbf{A 3 5}$ & $\mathbf{A 3 6}$ \\
\hline$C R$ & $2.1 \pm 0.2$ & $2.1 \pm 0.2$ & $4.7 \pm 0.5$ & $4.4 \pm 0.6$ \\
$P V$ & $4.5 \pm 0.7$ & $4.4 \pm 0.6$ & $3.7 \pm 0.4$ & $5.1 \pm 0.7$ \\
\hline
\end{tabular}

options for the further partitioning of cortical interneurons. In the cortex, calretinin and parvalbumin, are expressed exclusively in interneurons and characterize two subpopulations that do not overlap and differ significantly in morphology, synaptic contacts, and functional properties. In this study, CR and PV expressing neurons were selected for more detailed stereological and densitometric analysis in PRC and RSC in order to compare the inhibition system of both domains and to help clarify their different functions. A detailed analysis of $\mathrm{CR}+$ and $\mathrm{PV}+$ neurons in these areas has not yet been performed (Druga 2009) and only recently our results have been published (Barinka et al. 2012, Salaj, Druga et al. 2015).

A major finding of our study is the higher $\mathrm{CR}+$ neuron concentration and higher $\mathrm{CR}$ density in the PRC compared to RSC as well as to surrounding neocortical areas. In contrast, PV + neurons and PV density were similar in both investigated areas and did not differ significantly from surrounding neocortical regions, with the exception of A35 where the PV density and the percentage of PV + neurons were lower, see Fig. 2 and Table $1 \mathrm{~B}$ and Table 2.

\section{Incorporating both types of neurons into cortical circuits}

$\mathrm{PV}+$ neurons are a significant population of local inhibitory (GABAergic) interneurons and represent about $25 \%$ of all GABAergic neurons. They predominate in III. and IV. cortex layer. Their morphological correlates are basket cells, the fast-spiking interneurons that make perisomatic "basket" terminals on other cells, and chandelier cells, which target the axon initial segment of pyramidal cells (Tremblay, Lee et al. 2016). Their axonal ramification is oriented mainly horizontally. In many cortical regions, in addition to chemical synapse also electrical synapse in $\mathrm{PV}+$ neurons have been demonstrated. In neocortical formations, PV neurons are under the synaptic influence of spiny stellate cells and under the influence of excitatory thalamocortical projection. Inhibitory effects of $\mathrm{PV}+$ neurons are predominantly transmitted to excitatory pyramidal and spiny stellate cells. The effect of excitatory thalamocortical projection is transferred from $\mathrm{PV}+$ interneurons to excitatory interneurons (spiny stellate cells) and pyramidal neurons, which are subsequently inhibited. Based on many experimental data obtained both in vivo and in vitro, $\mathrm{PV}+$ neurons can be considered as the primary and very powerful inhibitory cortical mechanism responsible for perisomatic and, to a lesser extent, axo-axonal inhibition of efferent cortical neurons. The results obtained by the quantitative analysis in this study suggest that this is also the case in PRC and RSC (Barinka et al. 2012, Salaj et al. 2015, Winer et al. 2005, Hirsch and Martinez 2006, Druga 2009).

$\mathrm{CR}+$ neurons differ in many respects from PVexpressing neurons. Most $\mathrm{CR}+$ neurons exhibit bipolar morphology of the somatodendritic domain, multipolar morphology is uncommon. Axonal ramification is oriented vertically in bipolar neurons, whereas in multipolar neurons is oriented rather horizontally.

Synaptic contacts come from many sources, including excitatory cortical neurons, inhibitory cortical interneurons, and many subcortical structures (thalamus, serotoninergic, dopaminergic, noradrenergic and cholinergic projections). Axonal terminations were demonstrated predominantly on dendrites of other interneurons, including $\mathrm{CR}+$ neurons.

Bipolar CR neurons had much higher connection probability to multipolar CR neurons than to any other neuronal type, followed by other interneuronal types (including other bipolar CR cells) and pyramidal cells coming last (Tremblay et al. 2016). CR + neurons belong to the category of "non-fast spiking neurons" which generate a rhythmic activity that is irregular in nature or adapting bursting.

$\mathrm{CR}+$ neurons have inhibitory effects on other inhibitory neurons and thus inhibit the inhibition of pyramidal neurons. Recently, it is argued that $\mathrm{CR}+$ neurons are among the elements with complex functions that are part of all important mechanisms supporting neuronal functions, including circulatory and metabolic control (Cauli, Zhou et al. 2014, Gonchar and Burkhalter 1997, Zaitsev et al. 2005, Druga 2009).

\section{PRC feature}

Defining PRC features emphasizes perception 
and object identification, object resolution, and object similarity evaluation. Basically, changes in neuronal activities are in the "WHAT" question. In addition, the PRC is considered to be a source of sensory information for the lateral entorhinal region (LEC), which is functionally similarly oriented. The PRC is probably not involved in the process of identifying an object in space. (Deshmukh et al. 2012).

In addition to these functions, the PRC is considered a transient neo-allocortical region that has a suppressive effect on signal transmission between neocortical formation and hippocampus. Analysis of signal propagation from neocortex via PRC to entorhinal cortex (EC) showed a very low probability of transmission between PRC and EC. PRC can therefore be considered as a filter that significantly restricts bidirectional signal transfer between neocortex and hippocampus (de Curtis and Pare 2004). To explain this phenomenon, these authors assume a specific inhibitory mechanism whose substrate is probably GABAergic interneurons expressing calcium-binding proteins in superficial layers of PRC. We can confirm this assumption. The PRC inhibitory mechanism consists of the basic population of PV + neurons, which in PRC is not very different from the surrounding neocortex and high concentration of $\mathrm{CR}+$ neurons. Perisomatic and axo-axonal inhibition by $\mathrm{PV}+$ neurons has a suppressive effect on the activity of pyramidal neurons in a given cortical region, but is probably not sufficient to suppress the activity transfer in the PRC. However, to explain the effects of CR + neurons, it is necessary to know the effect of this population on the activities of cortical microcircles in the PRC. However, the structure of these circuits is not yet known.

\section{RSC function}

In a number of functional experiments, RSC participation has been repeatedly demonstrated for space navigation and spatial memory. The RSC integrates spatial environmental characteristics (allothetic) with information that monitors the movement of the organism (idiothetic). When discarding allothetic information, important information from proprioceptors, vestibular receptors and somatosensory information is important for navigation. Idiothetic information is transported to the hippocampus through the parietal, entorhinal and RSC cortical regions (Elduayen and Save 2014).

There are functional differences between the granular and dysgranular regions of the RSC. The granular region is involved in spatial learning and memory in both light and darkness, while the dysgranular region is activated only during memory tasks in the light. The granular region contributes to spatial learning and navigation using information from the external and internal environment of the organism, while the dysgranular region is selectively activated in environmental information tasks (e.g., remote visual stimuli) (Pothuizen et al. 2009). However, a number of experimental results suggest a significant role for RSC in addressing spatial navigation tasks in the elimination of visual stimuli (in the dark) (Cooper et al. 2001, Whishaw et al. 2001).

\section{Conclusions}

The present study summarizes the results of our work aimed at studying the inhibition system in two cortical regions - perirhinal and retrosplenial, which form the transition between neocortex and hippocampal formation. The analysis was focused on two populations of cortical inhibitory (GABAergic) interneurons expressing calcium-binding proteins Parvalbumin and Calretinin. Both study populations of interneurons are defined in the literature in terms of somatodendritic morphology, functional properties and involvement in cortical micro-circles.

Our analysis was focused on the laminar distribution of these interneurons, their somatodendritic morphology, their density and the optical density of parvalbumin and calretinin in the perirhinal and retrosplenial cortical region.

Qualitative and quantitative analysis was performed on the brains of eight adult Wistar rats. After a qualitative analysis of the material, both selected areas were processed by a stereological and densitometric method. Thus, data on the density of CR and PV positive neurons and data on the density of both proteins in studied cortical regions were obtained.

Densitometric analysis showed higher values of the optical density of calretinin and lower values of parvalbumin in the perirhinal area compared to the surrounding neocortex. Similarly, a higher density of calretinin-positive neurons was demonstrated. In contrast, in the retrosplenial region, higher values of optical density of parvalbumin and lower values of calretinin were shown. In the retrosplenial region is the lower density of calretinin and parvalbumin positive neurons.

Although retrosplenial and perirhinal areas are 
similar in some cytoarchitectonical respects, significant differences in the distribution of interneuronal populations have been demonstrated. The high concentration of calretinin and the high values of calretinin-positive neurons in the perirhinal region may be the substrate of the mechanism responsible for suppressing the transfer of neuronal activities from the neocortex to the hippocampus through the perirhinal and entorhinal cortical regions. Above mentioned differences in expression of $\mathrm{CR}$ and $\mathrm{PV}$ maybe also responsible for the functional differences between PRC and RSC. But without more detailed analysis of microcircuits in which are PV and CR neurons incorporated there differences cannot be more precisely defined.

\section{Conflict of Interest}

There is no conflict of interest.

\section{References}

BARINKA F, DRUGA R: Calretinin expression in the mammalian neocortex: a review. Physiol Res 59: 665-677, 2010. https://doi.org/10.33549/physiolres.931930

BARINKA F, SALAJ M, RYBAR J, KRAJCOVICOVA E, KUBOVA H, DRUGA R: Calretinin, parvalbumin and calbindin immunoreactive interneurons in perirhinal cortex and temporal area $\mathrm{Te} 3 \mathrm{~V}$ of the rat brain: qualitative and quantitative analyses. Brain Res 1436: 68-80, 2012. https://doi.org/10.1016/j.brainres.2011.12.014

BRODMANN K: Vergleichende Lokalisationslehre der Grosshirnrinde in ihren Prinzipien dargestellt auf Grund des Zellenbaues. Leipzig: Barth, 1909.

BURWELL RD: The parahippocampal region: corticocortical connectivity. Ann NY Acad Sci 911: 25-42, 2000. https://doi.org/10.1111/j.1749-6632.2000.tb06717.x

BURWELL RD: Borders and cytoarchitecture of the perirhinal and postrhinal cortices in the rat. J Comp Neurol 437 : 17-41, 2001. https://doi.org/10.1002/cne.1267

BURWELL RD, AMARAL DG: Cortical afferents of the perirhinal, postrhinal, and entorhinal cortices of the rat. J Comp Neurol 398: 179-205, 1998. https://doi.org/10.1002/(SICI)1096-9861(19980824)398:2<179::AIDCNE3>3.0.CO;2-Y

BURWELL RD, AMARAL DG: Perirhinal and postrhinal cortices of the rat: interconnectivity and connections with the entorhinal cortex. J Comp Neurol 391: 293-321, 1998. https://doi.org/10.1002/(SICI)10969861(19980216)391:3<293::AID-CNE2>3.0.CO;2-X

BURWELL RD, WITTER MP, AMARAL DG: Perirhinal and postrhinal cortices of the rat: a review of the neuroanatomical literature and comparison with findings from the monkey brain. Hippocampus 5: 390-408, 1995. https://doi.org/10.1002/hipo.450050503

CAULI B, ZHOU X, TRICOIRE L, TOUSSAY X, STAIGER JF: Revisiting enigmatic cortical calretinin-expressing interneurons. Front Neuroanat 8: 52, 2014. https://doi.org/10.3389/fnana.2014.00052

COOPER BG, MANKA TF, MIZUMORI SJ: Finding your way in the dark: the retrosplenial cortex contributes to spatial memory and navigation without visual cues. Behav Neurosci 115: 1012-1028, 2001. https://doi.org/10.1037/0735-7044.115.5.1012

DE CURTIS M, PARE D: The rhinal cortices: a wall of inhibition between the neocortex and the hippocampus. Prog Neurobiol 74(2): 101-110, 2004. https://doi.org/10.1016/j.pneurobio.2004.08.005

DEL RIO MR, DEFELIPE J: Colocalization of calbindin D-28k, calretinin, and GABA immunoreactivities in neurons of the human temporal cortex. J Comp Neurol 369: 472-482, 1996. https://doi.org/10.1002/(SICI)10969861(19960603)369:3<472::AID-CNE11>3.0.CO;2-K

DESHMUKH SS, JOHNSON JL, KNIERIM JJ: Perirhinal cortex represents nonspatial, but not spatial, information in rats foraging in the presence of objects: comparison with lateral entorhinal cortex. Hippocampus 22(10): 2045-2058, 2012. https://doi.org/10.1002/hipo.22046

DRUGA R: Neocortical inhibitory system. Folia Biol (Praha) 55: 201-217, 2009.

ELDUAYEN C, SAVE E: The retrosplenial cortex is necessary for path integration in the dark. Behav Brain Res 272 : 303-307, 2014. https://doi.org/10.1016/j.bbr.2014.07.009 
FURTAK SC, WEI SM, AGSTER KL, BURWELL RD: Functional neuroanatomy of the parahippocampal region in the rat: the perirhinal and postrhinal cortices. Hippocampus 17: 709-722, 2007. https://doi.org/10.1002/hipo.20314

GABBOTT PL, BACON SJ: Local circuit neurons in the medial prefrontal cortex (areas 24a,b,c, 25 and 32 ) in the monkey: I. Cell morphology and morphometrics. J Comp Neurol 364: 567-608, 1996. https://doi.org/10.1002/(SICI)1096-9861(19960122)364:4<567::AID-CNE1>3.0.CO;2-1

GONCHAR Y, BURKHALTER A: Three distinct families of GABAergic neurons in rat visual cortex. Cereb Cortex 7: 347-358, 1997. https://doi.org/10.1093/cercor/7.4.347

HIRSCH JA, MARTINEZ LM: Circuits that build visual cortical receptive fields. Trends Neurosci 29: 30-39, 2006. https://doi.org/10.1016/j.tins.2005.11.001

KAWAGUCHI Y, KUBOTA Y: GABAergic cell subtypes and their synaptic connections in rat frontal cortex. Cereb Cortex 7: 476-486, 1997. https://doi.org/10.1093/cercor/7.6.476

KUBOTA Y, HATTORI R, YUI Y: Three distinct subpopulations of GABAergic neurons in rat frontal agranular cortex. Brain Res 649: 159-173, 1994. https://doi.org/10.1016/0006-8993(94)91060-X

MARKRAM H, TOLEDO-RODRIGUEZ M, WANG Y, GUPTA A, SILBERBERG G, WU DC: Interneurons of the neocortical inhibitory system. Nat Rev Neurosci 5: 793-807, 2004. https://oi.org/10.1038/nrn1519

PAXINOS G, WATSON C: The Rat Brain in Stereotaxic Coordinates, 6th ed. Oxford: Elsevier Academic Press, Amsterdam. 2007.

POTHUIZEN HH, DAVIES M, ALBASSER MM, AGGLETON JP, VANN SD: Granular and dysgranular retrosplenial cortices provide qualitatively different contributions to spatial working memory: evidence from immediate-early gene imaging in rats. Eur J Neurosci 30: 877-888. 2009. https://doi.org/10.1111/j.14609568.2009.06881.x

SALAJ M, DRUGA R, CERMAN J, KUBOVA H, BARINKA F: Calretinin and parvalbumin immunoreactive interneurons in the retrosplenial cortex of the rat brain: Qualitative and quantitative analyses. Brain Res 1627: 201-215, 2015. https://doi.org/10.1016/j.brainres.2015.09.031

TOLEDO-RODRIGUEZ M, BLUMENFELD B, WU C, LUO J, ATTALI B, GOODMAN P, MARKRAM H: Correlation maps allow neuronal electrical properties to be predicted from single-cell gene expression profiles in rat neocortex. Cereb Cortex 14: 1310-1327, 2004. https://doi.org/10.1093/cercor/bhh092

TOLEDO-RODRIGUEZ M, GOODMAN P, ILLIC M, WU C, MARKRAM H: Neuropeptide and calcium-binding protein gene expression profiles predict neuronal anatomical type in the juvenile rat. J Physiol 567(Pt 2): 401413, 2005. https://doi.org/10.1113/jphysiol.2005.089250

TREMBLAY R, LEE S, RUDY B: GABAergic Interneurons in the neocortex: from cellular properties to circuits. Neuron 91: 260-292, 2016. https://doi.org/10.1016/j.neuron.2016.06.033

VAN GROEN T, WYSS JM: Connections of the retrosplenial granular a cortex in the rat. J Comp Neurol 300: 593-606, 1990. https://doi.org/10.1002/cne.903000412

VAN GROEN T, WYSS JM: Connections of the retrosplenial dysgranular cortex in the rat. J Comp Neurol 315 : 200-216, 1992. https://doi.org/10.1002/cne.903150207

VAN GROEN T, WYSS JM: Connections of the retrosplenial granular b cortex in the rat. J Comp Neurol 463: 249-263, 2003. https://doi.org/10.1002/cne.10757

WEST MJ, SLOMIANKA L, GUNDERSEN HJ: Unbiased stereological estimation of the total number of neurons in the subdivisions of the rat hippocampus using the optical fractionator. Anat Rec 231: 482-497. 1991. https://doi.org/10.1002/ar.1092310411

VOGT BA: Structural organization of cingulate cortex: Areas, neurons and somatodendritic transmitter receptors. In: Neurobiology of cingulate cortex and limbic thalamus. BA VOGT, M GABRIEL (Eds.). Birhauser, Boston, pp. 19-70. 1993. https://doi.org/10.1007/978-1-4899-6704-6_2

VOGT BA, VOGT L, FARBER NB: Cigulate cortex and disease models. In: The Rat Nervous System. G PAXINOS (Ed.), 3th Edition Elsevier Academic Press, San Diego, pp. 705-727 London. 2004. https://doi.org/10.1016/B978-012547638-6/50023-7 
WHISHAW IQ, MAASWINKEL H, GONZALEZ CL, KOLB B: Deficits in allothetic and idiothetic spatial behavior in rats with posterior cingulate cortex lesions. Behav Brain Res 118: 67-76, 2001. https://doi.org/10.1016/S0166$\underline{4328(00) 00312-0}$

WINER JA, MILLER LM, LEE CC, SCHREINER CE: Auditory thalamocortical transformation: structure and function. Trends Neurosci 28: 255-263, 2005. https://doi.org/10.1016/j.tins.2005.03.009

WITTER MP, AMARAL DG: Hippocampal formation. In: Paxinos, G. (Ed.), The Rat Nervous System 3th Edition Elsevier Academic Press, San Diego, pp. 635-704 London. 2004. https://doi.org/10.1016/B978-0125476386/50022-5

ZAITSEV AV, GONZALEZ-BURGOS G, POVYSHEVA NV, KRONER S, LEWIS DA, KRIMER LS: Localization of calcium-binding proteins in physiologically and morphologically characterized interneurons of monkey dorsolateral prefrontal cortex. Cereb Cortex 15: 1178-1186. 2005. https://doi.org/10.1093/cercor/bhh218

ZAITSEV AV, POVYSHEVA NV, GONZALEZ-BURGOS G, ROTARU D, FISH KN, KRIMER LS, LEWIS DA: Interneuron diversity in layers 2-3 of monkey prefrontal cortex. Cereb Cortex 19: 1597-1615. 2009. https://doi.org/10.1093/cercor/bhn198 